\title{
INTRODUCTION TO THE SPECIAL ISSUE ON E-INFRASTRUCTURES FOR EXCELLENT SCIENCE: ADVANCES IN LIFE SCIENCES, DIGITAL CULTURAL HERITAGE AND CLIMATOLOGY
}

It is our pleasure to present this special issue of the scientific journal Scalable Computing: Practice and Experience. In this issue (Volume 19, No 2 June 2018), we have selected 14 papers which have gone through peer review and represent novel results in the fields of Life Sciences, Digital Cultural Heritage and Climatology, using state-of-the-art e-infrastructures, regionally integrated under the framework of the VI-SEEM project, and the related Virtual Research Environment (VRE). e-Infrastructures offer state-of-the-art IT resources which are the foundation that supports the scientific excellence in contemporary research. The VI-SEEM VRE integrates resources across all layers of the e-Infrastructure (networking, computing, data, software, user interfaces) to foster scientific excellence in selected fields and encourage cross-disciplinarity as well.

In the content of this special issue the papers are ordered thematically in 4 groups: Climatology (5 papers), Life Sciences (3 papers), Digital Cultural Heritage (2 papers) and Tools and Services (4 papers).

Climatology. The first paper presents an online interactive platform that aims to provide weather information about Armenia by integrating observations, model and satellite data. The topic is interesting from the practical point of view and might be very useful, especially for meteorologists.

The second papers studies the effect of the dust on climate in the Caucasus region, with a specific focus on Georgia, using the Regional Climate Model RegCM interactively coupled to a dust model. The simulations cover the period 1985-2014 encompassing most of the Sahara, the Middle East, the Great Caucasus with adjacent regions.

The third paper provides insight in the performances of wind simulations for high resolution models of the terrain. The presented results rationalize the possibility to run in reasonable time high resolution models, while showing that the impact of turbulence does not have significantly increases the computing requirement.

The fourth paper presents adaptation and tuning of the RegCM model for the Balkan Peninsula and Bulgaria and development of a methodology able to predict possible changes of the regional climate for different global climate change scenarios and their impact on spatial/temporal distribution of precipitation, hence the global water budgets, to changes of the characteristics and spatial/temporal distribution of extreme, unfavorable and catastrophic events.

The fifth paper presents comparison of two approaches (static and dynamical) used to compute the vibrational spectra of two conformers of the free formic acid molecule. The topic is interesting within the context of the atmospheric chemistry research field and it is of sufficient importance regarding the vibrational spectroscopic data and induced temperature effects of intramolecular motions.

Life Sciences. The manuscript from Astsatryan et al. describes a platform, which consists of data repository and workflow management services for Molecular Dynamics simulations. The platform focuses on an interactive data visualization workflow service as a key to perform more in-depth analyzing of research data outputs.

The manuscript from Bigovic et al. describes the organic synthesis of three enol carbonate derivatives and the analysis of their interactions with T4 lysozyme L99A/M102Q using molecular dynamics (MD) simulations. The results obtained by different software packages are discussed.

The manuscript from Koteska et al. describes a semi-empirical Molecular Dynamics study of irinotecan, a colon cancer drug, using the atom-centered density matrix propagation approach. The described methodology was used to study the structure, dynamics, and rovibrational spectrum of irinotecan.

Digital Cultural Heritage. The paper of Charalambous and Artopoulos presents the deployment of the Clowder CMS system and the development of extraction services to handle, manage and automatically process Digital Cultural Heritage data in order to enable virtual collaboration for research in the South East and Eastern Mediterranean region. Technical descriptions of the system are given and some results are provided.

In the paper of Elfarargy and Rizq a software system called Virtual Museum Framework (VirMuF), which is a set of tools that can be used by non-developers to easily create and publish $3 \mathrm{D}$ virtual museums in a very short time is presented. VirMuF is an open-source and teams including software developers can further extend VirMuF to fit their needs. 
Software Tools and Services. Dimitrov and Stoyanov present the Data Discovery Service supporting the VI-SEEM project Virtual Research Environment - VRE. The solution is based on an open source platform with special customization regarding the data harvesting methods from diverse data sources and updating the available content so that the users will seamlessly access all the data from a single point.

The paper of Golubev et al. addresses the problems of optimization of medical image storing and secure access, using the DICOM system. Based on the Moldova DICOM Network architecture, the system enables distributed search, and transportation of DICOM images. Additionally, several optimization problems are addressed by the authors, along with the integration challenges within the VI-SEEM VRE.

In the paper of Mishev et al. the design, requirements and implementation of a federated virtual research environment, based on the service orientation paradigm, offering anything as a service solutions, have been considered. The challenges of the service management implementation focusing on interoperability by design and service management standards have been discussed.

The manuscript of Vudragovic et al. gives an extensive insight of the development and implementation of the DREAM dust model (DREAMCLIMATE service). Additionally, a use-case study of the premature mortality due to the desert dust in the North Africa - Europe - Middle East region for the 2005 obtained by the application of this model is presented, justifying the model and the applicability of the service itself.

We would like to thank all those who kindly contributed to this Special Issue: the authors who submitted their papers, reviewers for their help and proposed improvements, especially to Dr. Zoe Cournia, Dr. Theodoros Christoudias and Dr. George Artopoulos for their valuable remarks and suggestions. Our special gratitude is for the Editor-in-Chief, Professor Dana Petcu, for her constant support.

Aneta Karaivanova, IICT-BAS, Bulgaria

Anastas Mishev, UKIM, FYR of Macedonia 